\title{
FREQUENCY ENTRAINMENT IN A SELF-OSCILLATORY SYSTEM UNDER PARAMETRIC EXCITATION
}

\author{
Hoang Van Da - NguYen Thac Sy \\ Hanoi University of Mining and Geology
}

\begin{abstract}
In the present paper, the frequencies for appearing the entrained oscillations in a self-oscillatory system under the parametric excitation, have been determined.

The almost periodic oscillation which develops from the entrained oscillation when the driving frequency is given just outside the region of entrainment also have been investigated by the method of the phase plane.
\end{abstract}

\section{Introduction}

The phenomenon of frequency entrainment occurs when a periodic force is applied to a system whose free oscillation is of the self-excited type [1]. The entrainment of frequency still occurs in the interaction between self-excited oscillation and parametric one [2]. In the present paper the region of entrainment for one of such systems will be examined and a numerical analysis will be carried out to study the almost periodic oscillation which occurs just outside the region of entrainment.

\section{Entrained oscillation}

The system under consideration is governed by the equation:

$$
\ddot{z}+z-\varepsilon \beta\left(1-\gamma z^{2}\right) \dot{z}-\varepsilon \alpha z \cos \nu t=0,
$$

where $\beta, \alpha, \nu, \gamma$ are constants and $\varepsilon$ is a small parameter. When $\alpha$ is equal to zero, equation (2.1) is a classical Van der Pol's equation and the amplitude of stable stationary oscillation is:

$$
z_{0}=\sqrt{\frac{4}{\gamma}}
$$

When $\beta$ is equal to zero, the equation (2.1) describes a parametric oscillator. When a half of the driving frequency is in the neighbourhood of the natural frequency of the system:

$$
\left(\frac{\nu}{2}\right)^{2}=1+\varepsilon \Delta
$$


one may expect an entrained oscillation of the form:

$$
\begin{aligned}
& z=a \sin \frac{\nu}{2} t+b \cos \frac{\nu}{2} t \\
& \dot{z}=a \frac{\nu}{2} \cos \frac{\nu}{2} t-b \frac{\nu}{2} \sin \frac{\nu}{2} t .
\end{aligned}
$$

By using the averaging method [2] we obtain the following equations for $a$ and $b$ :

$$
\begin{aligned}
& \dot{a}=\frac{\varepsilon}{\nu / 2}\left[\frac{1}{2} \Delta b+\frac{1}{4} \alpha b+\beta \frac{\nu}{2}\left(\frac{1}{2} a-\frac{1}{8} a \gamma\left(a^{2}+b^{2}\right)\right)\right], \\
& \dot{b}=\frac{\varepsilon}{\nu / 2}\left[\frac{1}{2} \Delta a-\frac{1}{4} \alpha b+\beta \frac{\nu}{2}\left(\frac{1}{2} b+\frac{1}{8} b \gamma\left(a^{2}+b^{2}\right)\right)\right] .
\end{aligned}
$$

Introducing new variables:

$$
x=\frac{a}{z_{0}} ; \quad y=\frac{b}{z_{0}} ; \quad \sigma=-\frac{\Delta}{\nu / 2}
$$

and approximating $\nu / 2$ by unity lead the equations (2.4) to the form:

$$
\begin{aligned}
& \dot{x}=\frac{\varepsilon}{2}\left[-\sigma y+\frac{1}{2} \alpha y+\beta x\left(1-x^{2}-y^{2}\right)\right], \\
& \dot{y}=\frac{\varepsilon}{2}\left[\sigma x+\frac{1}{2} \alpha x+\beta y\left(1-x^{2}-y^{2}\right)\right] .
\end{aligned}
$$

The stationary solution $x_{0}, y_{0}$ of the equations (2.6) are determined by:

$$
\begin{array}{r}
\left(-\sigma+\frac{1}{2} \alpha\right) y_{0}+\beta x_{0}(1-A)=0 \\
\left(\sigma+\frac{1}{2} \alpha\right) x_{0}+\beta y_{0}(1-A)=0
\end{array}
$$

where $A=x_{0}^{2}+y_{0}^{2}$.

Eliminating $x_{0}, y_{0}$ from (2.7) gives:

$$
A\left(\sigma^{2}+\beta^{2}(1-A)^{2}-\frac{1}{4} \alpha^{2}\right)=0 .
$$

It is readily seen that the trivial solution is $A=0$ and the non-trivial solutions are determined by:

$$
\left(C_{0}\right): \quad \sigma^{2}+\beta^{2}(1-A)^{2}-\frac{1}{4} \alpha^{2}=0
$$




\section{Region of entrainment}

We use the variational equations in $\delta x$ and $\delta y$ to study the stability of the stationary oscillations obtained from (2.6):

$$
\begin{aligned}
& \delta \dot{x}=e_{1} \delta x+e_{2} \delta y, \\
& \delta \dot{y}=e_{3} \delta x+e_{4} \delta y,
\end{aligned}
$$

where

$$
\begin{array}{ll}
e_{1}=\beta\left(1-3 x_{0}^{2}-y_{0}^{2}\right) ; & e_{2}=-\sigma+\frac{1}{2} \alpha-2 \beta x_{0} y_{0}, \\
e_{3}=\sigma+\frac{1}{2} \alpha-2 \beta x_{0} y_{0} ; & e_{4}=\beta\left(1-x_{0}^{2}-3 y_{0}^{2}\right) .
\end{array}
$$

In order to classify the singularities, we calculate the quantities:

$$
\begin{aligned}
& p=e_{1}+e_{4}=2 \beta(1-2 A), \\
& q=e_{1} e_{4}-e_{2} e_{3}=\beta^{2}(1-A)(1-3 A)+\sigma^{2}-\frac{1}{4} \alpha^{2}+2 \alpha \beta x_{0} y_{0} .
\end{aligned}
$$

Multiplying the first equation of (2.7) by $x_{0}$ and the second one by $y_{0}$ then adding them give:

$$
\alpha x_{0} y_{0}=-\beta(1-A) A .
$$

Consequently, $q$ can be rewritten in the form:

$$
\begin{aligned}
& q=\beta^{2}(1-A)(1-5 A)+\sigma^{2}-\frac{1}{4} \alpha^{2}, \\
& p^{2}-4 q=4 \beta^{2}(2-A) A-4\left(\sigma^{2}-\frac{1}{4} \alpha^{2}\right) .
\end{aligned}
$$

The equations of the separatrices in the $A-\sigma$ plane are:

$$
\begin{array}{ll}
\left(C_{1}\right): p=0 & \text { or } A=\frac{1}{2}, \\
\left(C_{2}\right): q=0 & \text { or } \beta^{2}(1-A)(1-5 A)+\sigma^{2}-\frac{1}{4} \alpha^{2}=0, \\
\left(C_{3}\right): p^{2}-4 q=0 & \text { or } \quad \beta^{2}(2-A) A-\sigma^{2}+\frac{1}{4} \alpha^{2}=0 .
\end{array}
$$

These separatrices are shown in Fig. 1 for the values

$$
\varepsilon=0.1 ; \quad \beta=1 \text { and } \alpha=1 .
$$

It is readily verified that the ellipse $\left(C_{2}\right)$ is tangent to the ellipse $\left(C_{3}\right)$ at the two points lying on the line $\left(C_{1}\right)$. 
The nature of the different areas, which are separatrices by the bounds, is easily determined as:

For positive values of $q$, that is, in the interior of the ellipse $\left(C_{2}\right)$ we have saddle points $S$.

For negative values of $q$, that is, in the exterior of the ellipse $\left(C_{2}\right)$ we have

- For the case $p>0(A<1 / 2)$ unstable nodes $U N$ if $p^{2}-4 q>0$ (in the interior of the ellipse $\left.\left(C_{3}\right)\right)$ or unstable focuses UF if $p^{2}-4 q<0$ (in the exterior of the ellipse $\left.\left(C_{3}\right)\right)$,

- From these results we can identify on the $A-\sigma$ plane the areas corresponding to different types of singularities (Fig. 1a) and easily determine the stability of stationary solutions.

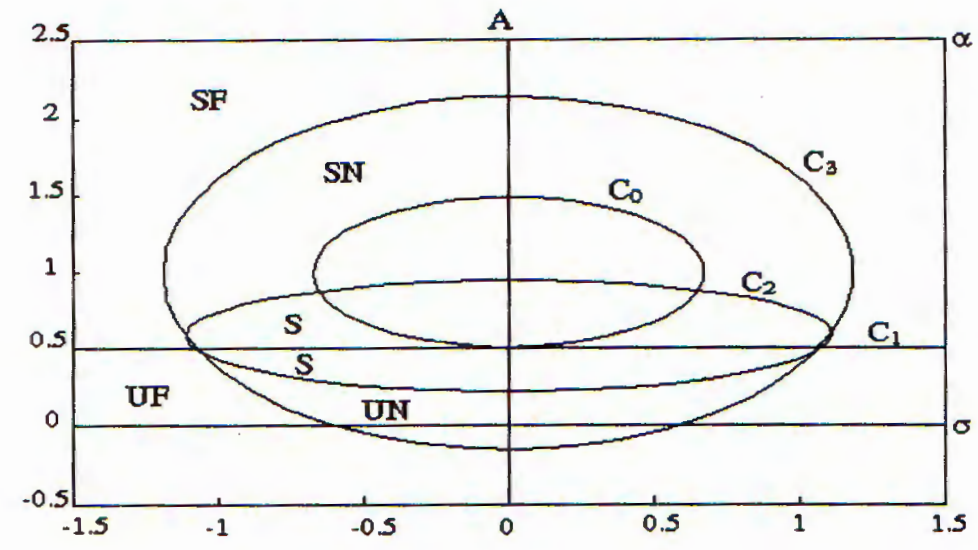

Fig. $1 a$

As mentioned above, the resonance curves (2.8) consist of two branches. The first one corresponding to trivial solutions is the line $A=0$. It is clear that they are unstable. The second branche $\left(C_{0}\right)$ is an ellipse described by the equation $(2.9)$ with center at $A=1, \sigma=0$ and two axes $\alpha$ and $\alpha / \beta$.

Differentiating both sides of (2.9) and setting $d \sigma / d A=0$ we obtain the locus of the vertical tangents of the resonance curve as:

$$
\beta^{2}(1-A)(1-3 A)+\sigma^{2}-\frac{1}{4} \alpha^{2}=0 .
$$

On the other hand, we try to find the locus of the points where the ellipse $\left(C_{2}\right)$ and the resonance curve $\left(C_{0}\right)$ intersect by adding their equations together. This leads to the same equation as (3.4). Thus the vertical tangencies of response curve occur at the intersection with the ellipse $\left(C_{2}\right)$. From here it follows that the upper part of the resonance curve $\left(C_{0}\right)$ limited by vertical tangents corresponds to the stable stationary oscillations. 
A similar analysis has been done for the cases in which half of the driving frequency is in the neighbourhood of $1 / 2$ or $1 / 3$ of the natural frequency of the system. Under this condition, no entrained oscillation is obtained. As a result, the frequency entrainment only occurs at the $1 / 2$ harmonic driving frequency, that is, when half of the frequency of parametric excitation is in the neighbourhood of the natural frequency of the system. In Fig. 1b the region of entrainment on the $\alpha-\nu$ plane is shown.

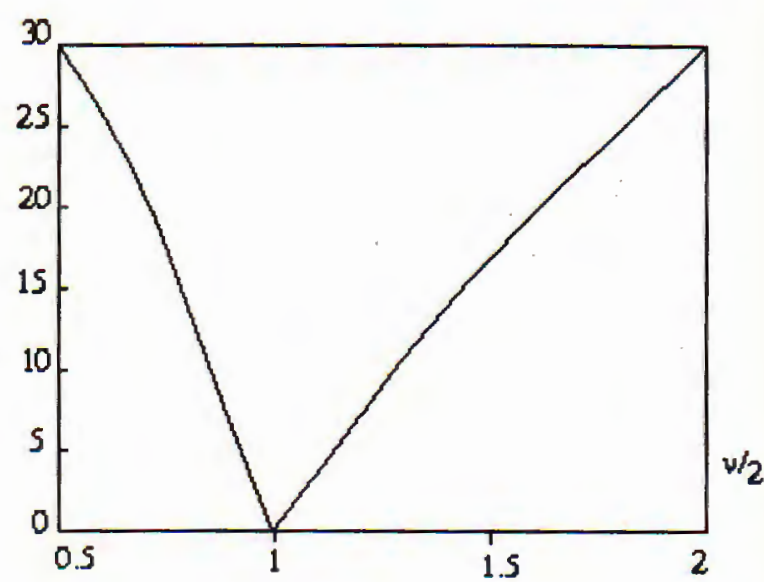

Fig. 16

\section{Almost periodic oscillation which develops from entrained oscilla- tion}

When the driving frequency is given just outside the region of entrainment, the subharmonic oscillation is no longer maintained in a stable state but is affected by amplitude and phase modulation due to the interaction with the self-excited oscillation. Consequently one may expect the occurrence of an almost periodic oscillation which can be expressed by $(2,3)$ and $(2.5)$ as:

$$
z=z_{0} x(t) \sin \frac{\nu}{2} t+z_{0} y(t) \cos \frac{\nu}{2} t,
$$

where $x(t)$ and $y(t)$ are slowly varying functions of $t$ and are to be found from the equations (2.6):

$$
\begin{aligned}
& \dot{x}=X(x, y), \\
& \dot{y}=Y(x, y) .
\end{aligned}
$$

To this end we consider the behaviour of integral curves defined by:

$$
\frac{d y}{d x}=\frac{X(x, y)}{Y(x, y)}
$$

in the $x-y$ plane. A numerical analysis was carried out by using the same parameters as in (3.3) and with $\gamma=1$. Typical examples in such a case are shown in Fig. 2a, 2b and $2 \mathrm{c}$. These figures show the integral curves under the conditions that the driving frequencies are given near the boundary of the region of entrainment $-0.5<\sigma<0.5$.

The integral curve for the case $\sigma=0.49$ is plotted in Fig. 2a, where we see 5 singularities. The unstable origin (1) corresponds to the trivial solution of the 
system. The singularities (4) and (5) are saddle curve $\left(C_{0}\right)$ in Fig. 1. The stable nodes (2) and (3) represent the entrained oscillation at the frequenciey $1 / 2 \nu$. It is clear that all initial conditions lead to this oscillation.

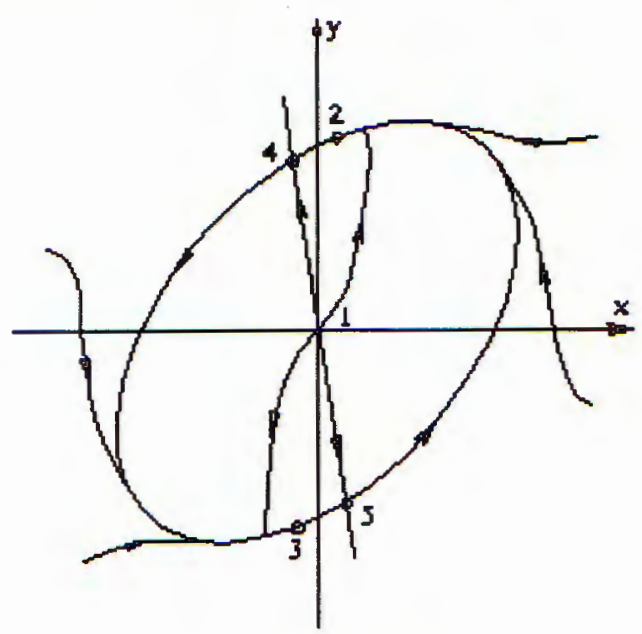

Fig. 2a

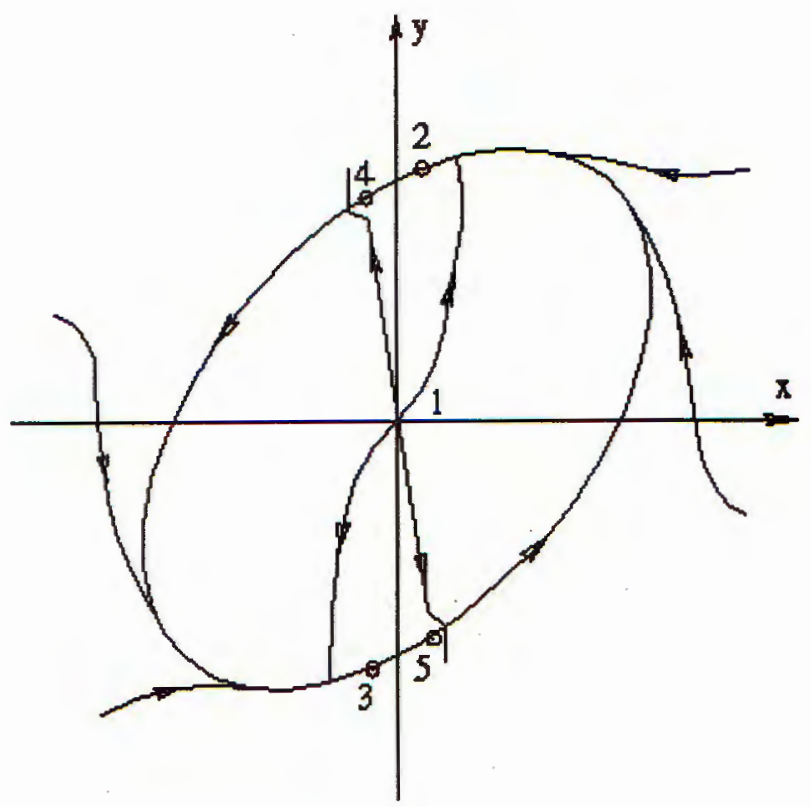

Fig. 26

At the boundary of entrainment $\sigma=0.5$ the coalescence of the stable node with the saddle takes place. In Fig. $2 \mathrm{~b}$ we see an unstable origin (1) and two unstable node-saddle points (2) and (3). The subharmonic oscillation is no longer sustained. Instead of this a limit cycle occurs, i.e. an almost periodic oscillation arises. 
When the detuning $\sigma$ is larger, $\sigma=0.51$, in Fig. $2 \mathrm{c}$ there is only an unstable origin and a limit cycle. That is. all initial conditions lead to the almost periodic oscillation. If the quantity $z$ in (4.1) is written in the form:

$$
z=r(t) \sin \left(\frac{\nu}{2} t+\theta(t)\right),
$$

the variation of the amplitude $r$ and phase $\theta$ are shown in Fig. 3. They vary slowly. The period required to complete one revolution along the limit cycle is $388.18 \ldots$ times the period of the parametric excitation. Since in the neighbourhood of the singular points from which the limit cycle has developed the representative point moves very slowly, most of the time the variation of the amplitude $r$ is small.

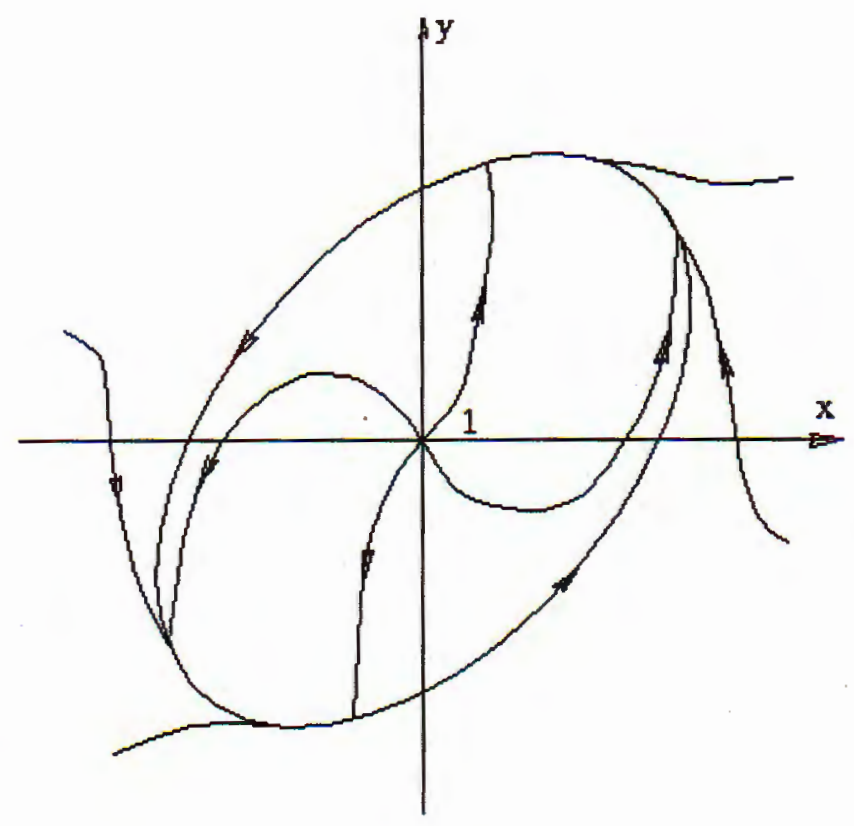

Fig. 2c

\section{Conclusion}

It was shown that there exists only one region of entrainment for the selfoscillatory system subjected to parametric excitation of the form (1.1) at the $1 / 2$ harmonic driving frequency, that is, when half of the frequency of the parametric excitation is in the neighbourhood of the natural frequency of the system. The occurrence of the almost periodic oscillation outside the region of entrainment and the transition between the entrained oscillation and the almost periodic oscillation have been examined by making use of the state plane method. 

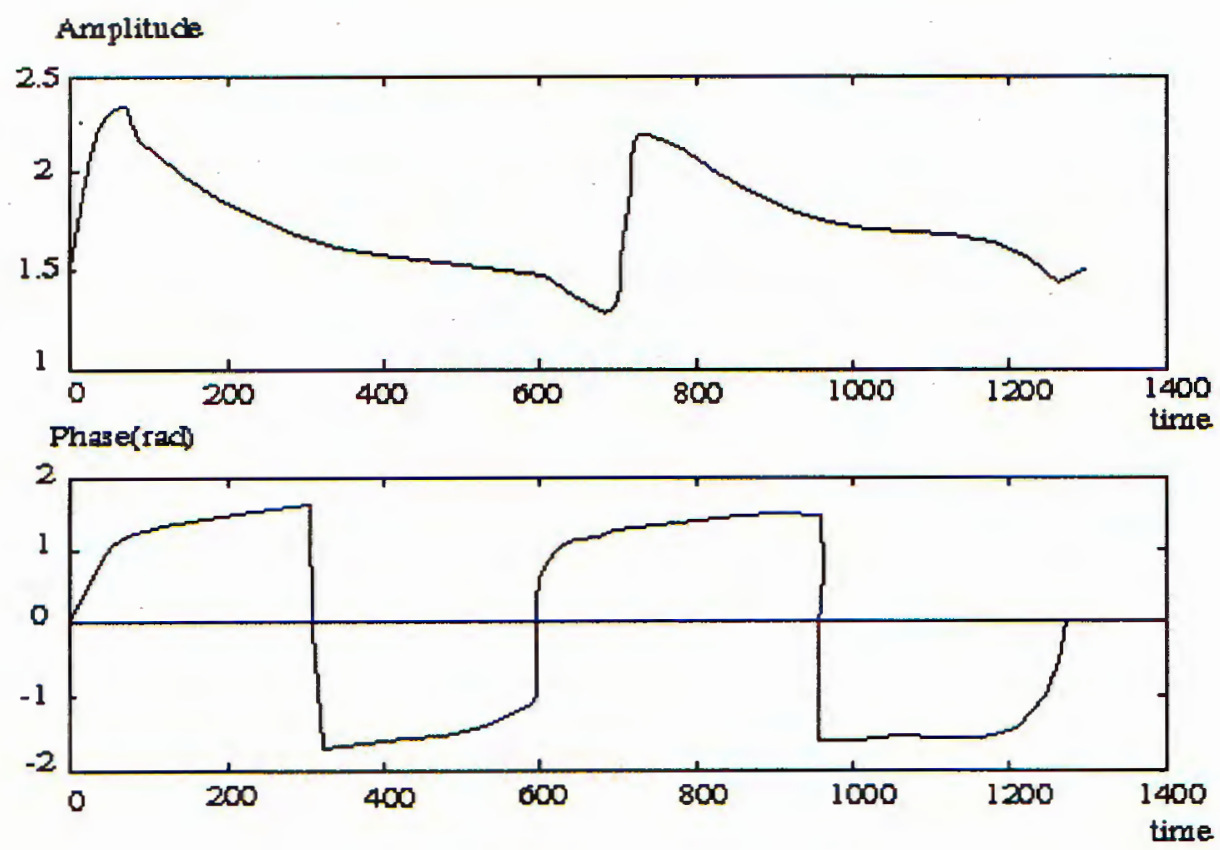

Fig. 3

This work is completed with financial support of the Council for Natural Sciences.

\section{REFERENCES}

1. Hayashi C. Nonlinear Oscillation in Physics System. McGraw-Hill, New York, 1964.

2. Yu. A., Nguyen Van Dao. Applied Asymtotic Methods in Nonlinear Oscillations. Hanoi, 1994.

3. Nguyễn Văn Đạo. Những phương pháp cơ bản của lý thuyết dao động phi tuyến. Nhà Xuất bản Đại học và Trung học chuyên nghiệp, Hà Nội, 1972.

Received September 18, 2002

DAO ĐộNG ĐỒNG Bộ TRONG HỆ TỰ DAO ĐộNG CHỊU KÍCH ĐỘNG THÔNG SỐ

Miền giá trị của các tần số kích động thông số để xuất hiện dao động đồng bộ trong một hệ tự động dao động chịu kích động thông số đã được xác định trong bài này. Các dao động tựa điều hòa phát sinh từ các dao động đồng bộ khi tần số kích động vượt qua ngoài miền giá trị trên cũng được khảo sát bằng phương pháp mặt phẳng trạng thái. 